\title{
Pedagogía en Teología: el aporte de Karl Rahner
}

\author{
FERNANDO BERRÍOS MEDEL \\ Pontificia Universidad Católica de Chile \\ fberriosm@uc.cl
}

\begin{abstract}
Resumen
La teología, que parte de la fe y encuentra su sentido en su servicio a ella en la especificidad de cada contexto histórico, no puede sino incluir en su propia finalidad la transmisión de esa fe. Karl Rahner, indiscutiblemente uno de los teólogos más importantes del siglo XX, se caracterizó, pese a la densidad y complejidad de su pensamiento, por su esfuerzo por hacer relevante el mensaje cristiano en la cultura moderna. Desde la comprensión de la impronta «antropológica» que el teólogo alemán intentó darle a su teología, el presente artículo pretende mostrar la luz que podemos encontrar en su método «trascendental» como camino de una auténtica pedagogía de la fe.
\end{abstract}

Palabras clave: pedagogía de la fe, método trascendental, Curso Fundamental de la Fe, espiritualidad y pastoral.

\section{Pedagogy in Theology: the contribution of Karl Rabner}

\begin{abstract}
Theology, that part of faith and finds it meaning in her service to the specificity of each historical context, can not but induce in his own end to the transmission of that faith. Karl Rahner, arguably one of the most important theologians of the twentieth century was characterized, despite the density and complexity of his thought, his effort to make the Christian message relevant in modern culture. From the understanding of imprinting «anthropological» than the German theologian tried to give his theology, this article tries to show the light to be found in his method "transcendental» as the path of an authentic pedagogy of faith.
\end{abstract}

Key words: pedagogy of faith, transcendental method, Foundations of Christian faith, spirituality and pastoral.

Doctor en Teología por la Universidad de Tübingen (Alemania), y postdoctorado en la misma universidad. Profesor de la Facultad de Teología de la PUC en el ámbito de la sistemática. Entre sus libros destacan: Teología del trabajo hoy. El desafio de un diálogo con la modernidad (1994) y Catolicismo social chileno. Desarrollo, crisis y actualidad (2009, en coedición con J. Costadoat y D. García). Algunos de sus artículos recientes son: "Von Balthasar y Rahner: vigencia de una interlocución teológica" (2009), "Una comunidad de discípulos misioneros. Líneas eclesiológicas de Aparecida" (2011); "Cien declaraciones de las Asambleas Plenarias del Episcopado chileno (1962-2010): Notas para una hermenéutica teológica” (2011).

Recibido: 22/Diciembre/2011 - Aceptado: 26/Enero/2012 


\section{Introducción: teología y transmisión de la fe}

La intuición de fondo en que se inscribe este tema es la conciencia creciente de que la teología no puede ser concebida tan solo como «la fe que busca comprender» (San Anselmo), por más ampliamente que queramos concebir ese «comprender», más allá de la dimensión cognitiva y racional del contenido de la doctrina cristiana. La teología tiene que ser también un aporte fundamental a la transmisión de la fe de la Iglesia, que es la matriz en la cual y para la cual ella existe. Tal vez en este sentido resulta más iluminadora la muy citada exhortación de 1Pe 3,15 a dar siempre «razón de nuestra esperanza». De lo que hay que «dar razón», según este texto, es de aquello que la fe nos permite esperar, dejando a la fe misma implícita. La intuición subyacente aquí es que la fundamentación del cristianismo tiene que ver ante todo con una experiencia y con las implicancias que ella tendrá necesariamente para el sentido del concreto existir del creyente.

En esta perspectiva es que tiene verdadero significado una reflexión sobre la relación entre pedagogía y teología. En lo que sigue se utilizará el concepto de pedagogía en un sentido muy amplio: como todo lo concerniente a los procesos de enseñanza-aprendizaje de los contenidos de la doctrina de la fe cristiana reflexionados por la teología, en cuanto ejercicio de transmisión de la experiencia subyacente a tal doctrina. Está de más decir que hay que desechar de partida que de lo que se trataría aquí es de reducir la teología a la catequesis, o viceversa. A este respecto resulta muy ilustrativa una reflexión que alguna vez hizo Karl Rahner en una carta fechada en München, 30 de mayo de 1964, dirigida a su discípulo y amigo Herbert Vorgrimler:

Se dice que mis clases son demasiado pesadas. Santo Dios, ¿qué puedo hacer? No puedo hacerlo de otro modo (...) ¿Qué hacer? En definitiva, las quejas me importan un bledo. Porque si de aquí sale un buen libro, es de más provecho para la Iglesia que edificar, como Guardini, a unos pocos centenares de gente necia. Eso es lo que pienso. ¿Qué opinas tú? (Vorgrimler, 1988: 255s).

Esta cita me parece interesante porque pone de manifiesto las dificultades concretas de un teólogo que intentaba dialogar con la cultura, pero a la vez con un grado tal de rigurosidad que lo apartaba de lo que él llamó el peligro de una «alta vulgarización» de la teología. El mismo Vorglimler ha descrito acertadamente el proceso que habría experimentado Rahner en ese intento. En un principio el teólogo jesuita 
se dirigió sobre todo a ambientes universitarios y a gente culta en general. Se esmeró en hablarles

...con el lenguaje de los entendidos en filosofía de aquellos años, con el lenguaje de Heidegger, de Jaspers, de los 'personalistas'... (Pero) en su edad madura este lenguaje se hizo más sencillo, más distendido... La razón no fue tan sólo que le llegaron las múltiples quejas sobre el lenguaje... Es que, además, ya no se sentía abrumado por el sentimiento de tener que precaverse, mediante innumerables circunlocuciones y oraciones secundarias, frente a las suspicacias de que sus afirmaciones fueran inconciliables con la tradición eclesial. Tuvo, además, que comprobar que ya no existía 'la' filosofía por la que estuvo tan marcado el lenguaje de sus primeros tiempos (Vorgrimler, 1988: 43).

Dentro de esos parámetros concretos, Rahner es, pues, una figura señera en lo que respecta a nuestro tema. Pero, como puede verse, no por haber sido un teólogo con un pensamiento de fácil comprensión, sino porque intentó establecer un acceso a los grandes temas de la teología en concordancia con ciertas intuiciones fundamentales de la cultura moderna. Aún más: es un teólogo que se hizo célebre ante todo por una determinada apuesta metodológica (cfr. Berríos, 2004). Y la metodología está a la base de toda pedagogía, si entendemos a aquélla en su sentido etimológico, es decir, como la reflexión o la toma de conciencia acerca del «camino a seguir» (met-odós) para alcanzar un determinado objetivo; en este caso, llegar a conocer en profundidad los fundamentos y las proyecciones de la fe y de la praxis cristianas. Esto se realizará solo si el método y el objetivo del conocimiento que se construye son efectivamente utilizados con fines educativos y no sólo teológicos, es decir, no con el mero propósito de crear saber experto.

Partiendo de estas consideraciones iniciales, hagamos ahora una breve aproximación al aporte de este gran teólogo al tema de la pedagogía en teología.

\section{El método antropológico trascendental como clave}

No viene aquí al caso exponer el método teológico de Rahner en todos sus contornos, sino más bien destacar su importancia como aporte a la dimensión pedagógica de la teología hoy. Por de pronto, me imagino que si un especialista en pedagogía se asomara a la obra de Rahner, le impresionaría, desde su punto de vista, el «centramiento antropológico» que caracteriza a su modo de pensar teológico. Me imagino que los 
pedagogos verían en él algo así como el ejemplo de un pensar «centrado en el sujeto», o que dirían que la teología de Rahner es una «teología constructivista», porque no parte por los grandes enunciados de la doctrina de la fe para luego deducir de ellos principios de validez universal, sino que, por el contrario, "parte desde el sujeto», que en este caso es el sujeto creyente.

Este es, pues, el punto decisivo del aporte pedagógico que debemos reconocer en la impronta antropológica que, sabemos, Rahner postuló que era necesario imprimir a toda la teología dogmática (Rahner, 1967: passim). En lo esencial, su intuición básica radica en su concepción «trascendental» de la revelación y de la gracia y, con ello, del acontecimiento global de la salvación (cfr. Berríos, 2002). Y, recordemos, el adjetivo «trascendental» tiene ante todo la connotación de que lo decisivo acontece no en la aprehensión del objeto del conocimiento y de la fe, considerado en su sola mismidad, sino en el sujeto y más exactamente, en las «condiciones de posibilidad» de tal conocimiento o encuentro; condiciones que se dan «a priori» en el sujeto cognoscente mismo. Pero se entiende, además, que se dan como necesaria «apertura», es decir, en orden a ese encuentro, preparado y gestado por la pura gratuidad del don de Dios. Por eso Rahner insiste en que en la relación:

...entre la subjetividad apriorística, trascendental, y el objeto del conocer (y de la libertad), el conocimiento de las condiciones apriorísticas de la posibilidad del conocimiento en el sujeto significa también un momento del conocimiento del objeto mismo (...)

Y por lo tanto:

Un planteamiento trascendental de la cuestión no es sólo una pregunta sobreañadida (zusäztlich) a la pregunta por el objeto que se presenta original y aposteriorística-empíricamente, sino que sólo en él el conocimiento del objeto mismo originario alcanza su plenitud (vollen Wesen). El conocimiento del sujeto cognoscente es, de por sí, siempre también un conocimiento de las estructuras metafísicas (en un sentido objetivo de 'trascendentales') del objeto mismo (Rahner, 1970: 98).

El método antropológico trascendental no es, pues, una pura «reducción antropológica» de la teología, como algunos han reprochado a Rahner, aunque sí implica los riesgos de asumir en la teología y en fidelidad a la Tradición, el punto de partida del pensar filosófico moderno y su «giro antropológico». Está fundado en una concepción epistemológica, es decir, en una determinada comprensión del conocimiento teológico que refleja, a su vez, una antropología; y tiene 
además una repercusión en lo pedagógico, que debe hacerse empero consciente y realizarse en una concreción siempre creativa.

\section{El Curso Fundamental de la Fe como figura paradigmática de una pedagogía teológica}

Como sabemos, el libro Curso Fundamental de la Fe (Grundkurs des Glaubens), no es propiamente una síntesis acabada del pensamiento teológico rahneriano, pero sí puede considerarse una presentación asentada de sus intuiciones fundamentales. El punto de partida de este «curso» es, precisamente, el creyente que busca vivir su fe y que descubre que para ello es necesario fundamentar esa misma fe ya vivida. Por último, está la convicción de que ello ha de hacerse en el contexto cultural de boy. He destacado tres elementos que son decisivos en el pensamiento teológico de Rahner y que encuentran un equivalente claro en el lenguaje actual de las ciencias de la educación: la vida de fe o experiencia cristiana (objetivo curricular); el fundamento de esa fe vivida (epistemología); y el hoy, porque el cristianismo, el discipulado de Jesús, es un dinamismo histórico que requiere de una permanente actualización (contexto). Es una tarea abierta y por lo tanto un proceso pedagógico - en un sentido amplio- permanente, es decir, que no es posible dar por terminado en ningún momento. Rahner habría acotado en este punto que todo ello es así, porque la salvación acontece como un don trascendental que empero está categorialmente mediado. El momento trascendental de la autocomunicación de Dios, "precisamente porque es permanente y está siempre dado, pertenece a esta historia y acontece en ella» (Rahner, 1999: 142).

Así, pues, eludir el triple desafío teológico y pedagógico al que nos hemos referido -el arraigo en la experiencia, la búsqueda del fundamento y la toma de conciencia sobre el contexto-, equivaldría, para Rahner, a abandonarnos, como cristianos y como Iglesia, a la irrelevancia de la fe en el acontecer histórico.

Especialmente interesante, como lo ha destacado Carlos Schickendantz (2004) en un artículo sobre el Grundkurs, es que, por todo lo dicho, en la perspectiva señalada por Rahner es más importante el «primer nivel de reflexión», que tiene que ver con el enfoque fundamental y con el género literario elegido. Es más importante que el «segundo nivel de reflexión», que tiene que ver con los contenidos concretos. 


\section{La vinculación entre teología, espiritualidad y pastoral}

Este punto es también muy importante para nuestro tema, tratándose de un teólogo claramente sistemático. Es sabido que su primer escrito fue sobre una temática espiritual (Rahner, 1924), así como su tesis de doctorado en teología. Más en general, la espiritualidad y la mística son temas transversales a toda su obra. Hasta sus últimos días, un tema recurrente en las reflexiones de Rahner fue el de la reductio in mysterium, en el sentido de una necesaria e íntima vinculación de teología y mística, como camino hacia el misterio absoluto de Dios (Rahner, 1970: 113-126). La «perspectiva trascendental» de su teología (transzendentaler Ansatz) no implica, como algunos han querido entender, una negación de la trascendencia divina, sino simplemente un modo concreto de entender el acontecimiento de la gratuita autodonación de Aquel semper maior.

Pero además no ha dejado de llamar la atención el hecho de que en el siglo XX algunos de los más importantes aportes a la teología práctica y sobre todo a la teología pastoral hayan venido «no de un teólogo práctico, sino del dogmático Karl Rahner» (Neumann, 1980: 21). Pensemos sobre todo en Sendung und Gnade. Beiträge zur Pastoraltheologie de 1959, y más tarde el célebre Handbuch der Pastoraltheologie (Rahner, 1964, 1966, 1968, 1969, 1972, 1995). Si revisamos estos textos, nos impresionará no sólo su rigurosidad sino también, lisa y llanamente, su alta complejidad. No se trata, ni mucho menos, de textos fáciles que desciendan a consejos prácticos para diseñar el plan pastoral de una parroquia. Y sin embargo, tienen una impronta claramente pastoral, si por ello se entiende una reflexión teológica fundada sobre la actualización de la misión de la Iglesia y, con ello, de la identidad misma eclesial, en el aquí y ahora de la cultura y de la sociedad. Se señala con esto una vital vinculación entre teología, espiritualidad y pastoral, que se relaciona a su vez con el siguiente aspecto de la teología rahneriana que quisiera destacar.

\section{El contenido del estudio teológico}

La adopción del método antropológico trascendental marca, por cierto, una cierta selección o priorización de los contenidos a abordar por la teología. Pero más importante, por cierto, es la perspectiva que ese método determina. Toda la teología de Rahner es una comprobación de este enunciado. No hay ningún gran tema de la teología que el jesuita alemán no haya integrado en su reflexión teológica trascendental. El 
tema, en cuanto objeto, no es lo decisivo, sino la mirada que podamos desplegar sobre y a partir de él, para iluminar la existencia de los cristianos contemporáneos.

En lo concreto, esta mirada comporta una intuición decisiva para, por ejemplo, la elaboración de los programas de los cursos teológicos hoy en día. Se hace necesario priorizar y seleccionar los contenidos, dependiendo de los alumnos; pero finalmente lo más importante no será eso, sino el modo de abordarlos, el punto de partida, la contextualización y los acentos que se pongan.

\section{Hacer teología en diálogo con la época y a la vez en fidelidad a la Tradición}

Rahner habló como teólogo a su época y utilizando, en gran medida, el lenguaje de su época. Al menos, como hemos visto, el lenguaje de la gente culta de su época. ¿Cómo lo haría hoy, si viviera en la Alemania, en la Europa y en el mundo globalizado de hoy? ¿Cuáles serían los equivalentes de las categorías filosóficas heideggerianas y kantianas de los que se valió en su momento?

En este punto hay que hacer notar el hecho de que la teología de Rahner no fue, como algunos podrían creer, un ejercicio de pura «traducción» del contenido de la fe cristiana en las categorías de dicho instrumental filosófico moderno, sino que lo que él intentó fue algo mucho más complejo, y que se expresa de un modo especial en su libro Geist in Welt de 1939: una interpretación de la metafísica tomista del conocimiento, a través de la relectura de Joseph Maréchal de la epistemología kantiana e iluminada por el pensamiento heideggeriano de los años veinte y los primeros de la década del treinta. Es decir, una asunción de los impulsos más importantes del pensamiento filosófico moderno, pero referido a lo mejor de la tradición tomista en la que el jesuita había sido formado, más allá de las limitaciones de la neoescolástica, que él mismo se encargaría de criticar.

En este intento Rahner alcanzó una síntesis personal muy característica, y toda su vida académica fue un proceso de profundización y de matización de esa síntesis. Pero él mismo reconoció en entrevistas al final de su vida, que después de un momento inicial no continuó su diálogo con la filosofía contemporánea. Al menos, por ejemplo, no siguió la evolución del pensamiento de Heidegger (Lehmann, 1994: 20). ¿Qué quedó entonces? Lo fundamental de su perspectiva trascendental, que él insistió en explicar ante todo como una perspectiva propiamente teológica, antes que filosófica o de otra índole (Rahner, 1967: 49-61). 


\section{Para seguir su ejemplo o recorrer su senda...}

Brevemente y a modo de síntesis, ¿qué habría que destacar como intuiciones importantes de Karl Rahner sobre el tema de la pedagogía en teología? Sugiero tres aspectos.

Primero, el partir siempre - en el diseño de los programas de los diversos cursos de teología, pero también al abordar en ellos cada tema en particular- desde los participantes. Con esto quiero decir: no sólo desde los estudiantes, sino también desde el profesor, con su historia y su contexto. Más exactamente: partir de lo que sabemos y de lo que no sabemos; no eludir ni siquiera los malentendidos o deformaciones acumuladas sobre la temática del curso. Pero también construir desde las riquezas que se tienen, en la mayoría de los casos, tal vez, sin haber tomado conciencia refleja de ello. Así, lo que está en nosotros, para bien o para mal, ayudará siempre a emprender un camino de aprendizaje y de reflexión más significativo y más relevante desde el punto experiencial. Asunto nada secundario, considerando que la teología se ejerce a partir de la fe, nunca sin ella, y en consecuencia, no al margen sino en el centro del camino de la salvación como acontecer histórico.

Segundo, evitar conscientemente el complejo de que la teología tiene que marcar a toda costa la diferencia con la espiritualidad. Si la teología nace de la fe de la Iglesia y se ordena a su servicio, entonces más bien uno de sus principales objetivos debe ser el aporte de una fundamentación sólida de la experiencia espiritual de esa fe. En esta intuición está el contenido nuclear de la muy citada reflexión de Rahner: «El cristiano del futuro será un místico o ya no será cristiano» (Man hat schon gesagt, daß der Christ der Zukunft ein Mystiker sei oder nicht mehr sei). (Rahner, 1980: 375). El teólogo alemán hace esta afirmación en el contexto de una rigurosa caracterización de la espiritualidad y de la mística como una actitud resuelta, audaz y en absoluto prescindente de los desafíos de la existencia histórica, sino, por el contrario, en el corazón mismo de esa experiencia.

Tercero, hacer siempre el ejercicio de abrirse a las preguntas que se plantean a cada tratado desde la pastoral. Destaco las palabras ejercicio, en el sentido de una búsqueda, y de una búsqueda compartida; preguntas, porque son más importantes que las supuestas respuestas (que suelen darse con excesiva premura) y porque son una concreción de ese espíritu de búsqueda del que hablamos; y por último, el concepto de pastoral, porque es necesaria una reivindicación de la teología pastoral en toda su seriedad y rigurosidad, y en esto el aporte de Rahner ha sido decisivo. Pero aquí hay que recorrer un camino arduo, que parte por no admitir la 
tentación de buscar las salidas fáciles e inmediatistas a problemas que son muy complejos y que plantean temas de fondo. En este aspecto, Rahner, y yo diría que la teología alemana en general, hasta hoy, son muy tajantes, porque reservan para la teología pastoral todos los temas que pueden tener alguna relación directa con la «autorrealización (Selbstvollang) de la Iglesia» en el mundo del hoy, y limitan a la dogmática, en especial a la eclesiología, el análisis de los temas que tienen que ver con su «esencia»y con la índole de su función en orden a la salvación. Pero eso es tema para otra discusión.

Visto en su conjunto, podemos concluir que el aporte del pensamiento teológico de Karl Rahner y de su método puede sernos de gran ayuda para enfocar el estudio de y la reflexión sobre los contenidos de la fe, a partir de la concreta situación en la que todos los creyentes estamos insertos y en función de las cuales estamos llamados a descubrir el sentido de nuestra existencia cristiana. Como el mismo jesuita lo dijo en su momento, el método antropológico-trascendental no abarca el todo del quehacer teológico ni debe entenderse como la única perspectiva posible para pensar la relación salvífica del Dios de Jesucristo con el ser humano (Rahner, 1970: 95.112), pero no es menos cierto que constituye uno de los más importantes aportes del siglo XX al desarrollo de la teología como búsqueda incesante de la comprensión integral de la fe y de la praxis del cristiano.

\section{REFERENCIAS}

-Berríos, F. (2002). Teología trascendental y praxis. Una reflexión desde el legado de Karl Rahner, Teologia y Vida (43), 467-502.

-Berríos, F. (2004). El método antropológico-trascendental de Karl Rahner como hermenéutica teológica del mundo y de la praxis, Teología y Vida (45), 411437.

-Lehmann, K. (1994). Philosophisches Denken im Werk Karl Rahners. En A. Raffelt (Hg.), Karl Rahner in Erinnerung (págs. 10-27). Düsseldorf: Patmos.

-Neumann, K. (1980). Der Praxisbezug der Theologie bei Karl Rabner. FreiburgBasel-Wien: Herder.

-Puntel, L. B. (1979), Zu den Begriffen «transzendental» und «kategorial» bei Karl Rahner. En H. Vorgrimler (Hg.), Wagnis Theologie. Erfahrungen mit der Theologie Karl Rahners (págs. 189-198). Freiburg i.Br.-Basel-Wien: Herder.

-Rahner, K. (1924). Warum uns das Beten not tut, Leuchtturm 18 (25) 10-11.

-Rahner, K. (1999), Grundkurs des Glaubens. Einführung in den Begriff des Christentums (original: 1976). Sämtliche Werke (Bd. 26, ed. por N. Schwerdtfeger - A. Raffelt, págs. 3-445), Düsseldorf-Freiburg i. Br.: Herder. 
-Rahner, K. (1999). E latere Christi: Der Ursprung der Kirche als zweiter Eva aus der Seite Christi des zweiten Adam. Eine Untersuchung über den typologischen Sinn von Joh 19,34. - Original mecanografiado inédito, Karl Rahner-Archiv, Innsbruck: I B 6. Recogida en Sämtliche Werke (Bd. 3, ed. por A. R. Batlogg/E.Farrugia/K.-H. Neufeld), Freiburg i.B.: Herder.

-Rahner, K. \& Schuster, H. (Eds.). (1964). Handbuch der Pastoraltheologie. (Vol. I) Freiburg: Herder.

-Rahner, K. \& Schuster, H. (Eds.). (1966). Handbuch der Pastoraltheologie. (Vol. II) Freiburg: Herder.

-Rahner, K. \& Schuster, H. (Eds.). (1968). Handbuch der Pastoraltheologie. (Vol. III) Freiburg: Herder.

-Rahner, K. \& Schuster, H. (Eds.). (1969). Handbuch der Pastoraltheologie. (Vol. IV) Freiburg: Herder.

-Rahner, K. \& Schuster, H. (Eds.). (1972). Handbuch der Pastoraltheologie. (Vol. V: Lexikon der Pastoraltheologie) Freiburg: Herder.

-Rahner, K. (1980). Elemente der Spiritualität in der Kirche der Zukunft. En K. Rahner, Schriften zur Theologie (Bd. XIV, págs. 368-381). Ensiedeln-ZürichKöln: Benziger.

-Rahner, K. (1959). Sendung und Gnade. Beiträge zur Pastoraltheologie. Innsbruck: Tyrolia.

-Rahner, K. (1967). Theologie und Anthropologie. En K. Rahner, Schriften zur Theologie. (Bd. VIII, págs. 43-65). Einsiedeln-Zürich-Köln: Benziger.

-Rahner, K. (1970). Überlegungen zur Methode der Theologie. En K. Rahner, Schriften zur Theologie (Bd. IX, págs. 79-126). Ensiedeln-Zürich-Köln: Benziger.

-Rahner, K. (1995). Sämtliche Werke. (Bd. 19, ed. por K. Neufeld) DüsseldorfFreiburg i.Br.: Herder.

-Schickendantz, C. (2004). El 'Curso Fundamental de la Fe' de Karl Rahner. Objetivo, génesis y significado de un 'experimento'. Teología y Vida (45), 137-156.

-Vorgrimler, H. (1988). Entender a Karl Rabner. Introducción a su vida y su pensamiento (M. Villanueva Salas, Trad.). Barcelona: Herder.

Sumario: Introducción: teología y transmisión de la fe; 1. El método antropológico trascendental como clave; 2. El Curso Fundamental de la Fe como figura paradigmática de una pedagogía teológica; 3. La vinculación entre teología, espiritualidad y pastoral; 4. El contenido del estudio teológico; 5. Hacer teología en diálogo con la época y a la vez en fidelidad a la Tradición; 6. Para seguir su ejemplo o recorrer su senda...; Referencias. 